\title{
Intratarsal Keratinous Cyst - An Emerging Entity
}

\author{
Fatemeh Rajaii Abdalhossein Ghafourian Charles G. Eberhart \\ Wilmer Eye Institute, Johns Hopkins University School of Medicine, Baltimore, Md., USA
}

\section{Key Words}

Intratarsal keratinous cyst · Tarsal cyst · Eyelid cyst · Keratinous cyst · Eyelid lesion · Tarsal lesion

\begin{abstract}
We report two cases of recurrent intratarsal keratinous cysts of the Meibomian gland, a recently described lesion thought to have been previously misdiagnosed. The correct diagnosis can be made based on histopathologic features alone, although expression of cytokeratin (CK) $5 / 6$ in the cyst epithelium is a specific marker of these lesions. Recognition of these lesions, and distinguishing them from other intratarsal cysts that do not recur, is important to guide definitive therapy via full-thickness excision rather than simple incision and drainage.
\end{abstract}

(C) 2013 S. Karger AG, Basel

\section{Case 1}

A 76-year-old male with a history of a car accident in December 2008, with the subsequent repair of a ZMC fracture, a left paralytic ectropion repair using ear cartilage in March 2009, a left upper eyelid wedge resection for floppy eyelid syndrome in December 2009, left ptosis repair, blepharoplasty and the removal of a glass foreign body from the medial canthus in February 2010 presented with a complaint of left eye pain that had not changed since the car accident. Examination revealed a visual acuity of $20 / 25+1$ on the right, and pinhole visual acuity of 20/20 on the left. Additionally, he had Meibomian gland dysfunction and a tarsal cyst on the left upper eyelid at the site of the previous wedge resection. A wedge resection of the tarsal cyst was performed. Histologic analysis revealed a keratinous cyst in the tarsus, lined by stratified squamous epithelium featuring crenulations; the superficial layers of the epithelium were eosinophilic. There was keratinous material within the cyst as well as scarring and a chalazion surrounding the cyst (fig. 1a). There was not sufficient tissue to perform immunohistochemical analysis. 
Rajaii et al.: Intratarsal Keratinous Cyst - An Emerging Entity

\section{Case 2}

A 57-year-old Caucasian female with a history of bilateral upper and lower eyelid blepharoplasty presented to the clinic with a complaint of a recurrent left upper eyelid lesion that had been excised two years prior. Initially, the lesion had been present for about a year, had developed surrounding erythema, and had burst a few times, producing a white discharge. At that time the excised lesion had been identified as a benign epithelial cyst in the tarsus by pathology. After excision, the patient had relief until three months prior to presentation, when the cyst recurred, causing foreign body sensation in the left eye with blinking. Examination revealed pinhole visual acuity of 20/20 bilaterally. External examination was remarkable for acne rosacea of the upper and lower eyelids and mid face bilaterally as well as bilateral Meibomian gland dysfunction. On eversion of the left upper eyelid, there was a small, white, well-circumscribed, nodular lesion in the tarsus with inflammation of the surrounding conjunctiva (fig. 1b). The lesion was removed using a full-thickness excision of the conjunctival tarsus, and the defect was covered with an amniotic membrane patch graft.

Histopathologic examination revealed an intratarsal keratinous cyst lined by stratified squamous epithelium with a smooth luminal aspect, containing cord-like keratin lamellae. The epithelium lacked goblet cells and sebaceous cells; however, there were few clear cells present (fig. 1c, d). Immunohistochemistry revealed expression of pancytokeratin (AE1/AE3) in the cyst cuticle and lining (fig. 1e). The expression of AE1/AE3 was nonspecific and also seen in adjacent Meibomian gland ducts (data not shown). In addition, there was expression of cytokeratin (CK) 903 in the epithelium, cuticle, and contents, and expression of CK 5/6 in the cyst lining and contents, but not cuticle (fig. 1f, g; table 1). The sloughed keratinous material within the cyst strongly expressed CEA (fig. 1h), but we did not identify staining with this antibody in the normal Meibomian gland. The cyst was immunonegative for p16, CK 7, CK 18, CK 20, and EMA (table 1). The Ki67 expression index was very low.

\section{Discussion}

Acquired cystic lesions in the eyelid can arise from the sweat glands, such as apocrine and eccrine hidrocystomata, from pilosebaceous follicles, such as milia and tricholemmal cysts, or from the epidermis, such as epidermal inclusion cysts [1]. Intratarsal keratinous cysts are a recently described entity that arise from the Meibomian glands and are thought to be the third major cause of intratarsal swelling after chalazia and sebaceous cell carcinoma [2]. The incidence of these lesions is, however, unknown. The cysts are reported to have a variety of clinical appearances, including that of a white tarsal lesion as described above; multiple cysts may exist in a single eyelid [3]. Transconjunctival extrusion of the keratinous content has also been reported [4].

A similar lesion was initially described in the context of a 56-year-old patient with nevoid basal cell carcinoma syndrome; in that case, it was termed an epidermoid cyst of the eyelid and clinically consisted of a blue-colored lesion fixed within the tarsus [5]. Subsequent reports of lesions likely to be intratarsal keratinous cysts have not been in the context of cutaneous malignancy. Lucarelli et al. [6] reported three lesions, which they termed intratarsal epidermal inclusion cysts, in three 63- to 79-year-old patients; however, Jakobiec et al. [2] argue that these were likely also intratarsal keratinous cysts.

In a recent series of 6 cases reported by Jakobiec et al. [2], the lesions were characterized as intratarsal keratinous cysts. These arose in patients aged 31 to 76 years, and varied in clinical appearance from blue-gray to yellow-white to white nodules; all were non- 
Rajaii et al.: Intratarsal Keratinous Cyst - An Emerging Entity

inflamed and had discrete margins. The authors used immunohistochemical markers to characterize these lesions as distinct from normal epidermis or cutaneous epidermal cysts on the basis of the expression of CK 17 and CEA [2]. Furthermore, they found that while CK 17 expression is inconsistently seen in normal Meibomian acini and ducts, CEA staining is absent in the normal Meibomian structures, suggesting that CEA staining is the most specific marker of intratarsal keratinous cysts. Comparison of CK profiling by immunohistochemistry of one of the intratarsal keratinous cysts that we present to those described by Jakobiec et al. [2] reveals a somewhat altered pattern of CK 5/6, CK 7, and AE1/AE3 expression (table 2), suggesting that keratin expression in these lesions can vary. However, CEA expression is a consistent feature of the cyst that we examined and those reported previously by Jakobiec et al. [2].

In each of the cases that we present, there is a history of surgical trauma to the eyelids prior to intratarsal keratinous cyst formation. Lucarelli et al. [6] proposed that eyelid surgery or trauma may cause a blockage of the tarsal sebaceous ducts and eventual squamous metaplasia leading to the development of intratarsal keratinous cysts. A metaplastic mechanism, however, has been dismissed by Jakobiec et al. [2], who noted that the ducts and ductules of the Meibomian glands are composed of non-keratinizing squamous epithelium, and that there was no histological evidence of squamous metaplasia.

Given the variety of clinical appearances, intratarsal keratinous cysts may be mistaken for chalazia and other benign eyelid lesions. Unlike chalazia, however, simple drainage with excision of the capsule is not appropriate because of the tendency of these lesions to recur. Complete cyst excision with adjacent tarsectomy or full-thickness eyelid resection is recommended [2, 6]. The use of amniotic membrane or other graft material may be considered depending on the extent of tarsal conjunctiva that is involved. Accurate clinical and histopathologic identification is necessary to guide complete excision in order to prevent recurrence. CK expression profiling may be helpful in confirming the diagnosis; however, the histologic features alone can be diagnostic.

\section{Disclosure Statement}

The authors have no financial disclosures. C.G.E. is funded by Research to Prevent Blindness.

\section{References}

1 Font RL: Eyelids and lacrimal drainage system; in Spencer WH (ed): Ophthalmic Pathology: An Atlas and Textbook, ed 4. Philadelphia, Saunders, 1996, pp 2340-2345.

-2 Jakobiec FA, Mehta M, Iwamoto M, Hatton MP, Thakker M, Fay A: Intratarsal keratinous cysts of the Meibomian gland: distinctive clinicopathologic and immunohistochemical features in 6 cases. Am J Ophthalmol 2010;149:82-94.

-3 Kim HJ, Wojno TH, Grossniklaus HE: Multiple intratarsal keratinous cysts of the eyelid. Ophthal Plast Reconstr Surg 2012;28:e116.

-4 Gologorsky D, Jakobiec FA, Freitag SK: Transconjunctival elimination of keratin from an intratarsal Meibomian cyst. Ophthal Plast Reconstr Surg 2012 E-pub ahead of print.

5 Wolter JR, Mesaros MP: Epidermoid cyst of the eyelid in the nevoid basal cell carcinoma syndrome. J Pediatr Ophthalmol 1977;14:48-50.

-6 Lucarelli MJ, Ahn HB, Kulkarni AD, Kahana A: Intratarsal epidermal inclusion cyst. Ophthal Plast Reconstr Surg 2008;24:357-359. 
Rajaii et al.: Intratarsal Keratinous Cyst - An Emerging Entity

Table 1. CK expression profile of the intratarsal keratinous cyst described in case 2

\begin{tabular}{ll} 
CK & Staining \\
\hline AE1/AE3 & + \\
CK 903 & + \\
CK $5 / 6$ & + \\
CEA & + \\
p16 & - \\
CK 7 & - \\
CK 18 & - \\
CK 20 & - \\
EMA & - \\
\hline
\end{tabular}

- = Indicates no staining; + = indicates positive staining.

Table 2. Comparison of the CK expression profile of intratarsal keratinous cysts

\begin{tabular}{|c|c|c|c|c|c|c|}
\hline \multirow[t]{2}{*}{ CK } & \multicolumn{2}{|c|}{ Epithelium } & \multicolumn{2}{|l|}{ Cuticle } & \multicolumn{2}{|c|}{ Contents } \\
\hline & case 2 & $\begin{array}{l}\text { Jakobiec et al. } \\
\text { [2] }\end{array}$ & case 2 & $\begin{array}{l}\text { Jakobiec et al. } \\
\text { [2] }\end{array}$ & case 2 & $\begin{array}{l}\text { Jakobiec et al. } \\
\text { [2] }\end{array}$ \\
\hline CK 5/6 & + & ++ & - & - & ++ & ++ \\
\hline CK 7 & - & - & - & - & - & $+/-$ \\
\hline AE1/AE3 & - & + & + & + & + & + \\
\hline CEA & - & ++ & ++ & ++ & ++ & ++ \\
\hline
\end{tabular}

- = Indicates no staining; + = indicates positive staining; ++ = indicates robust positive staining. 

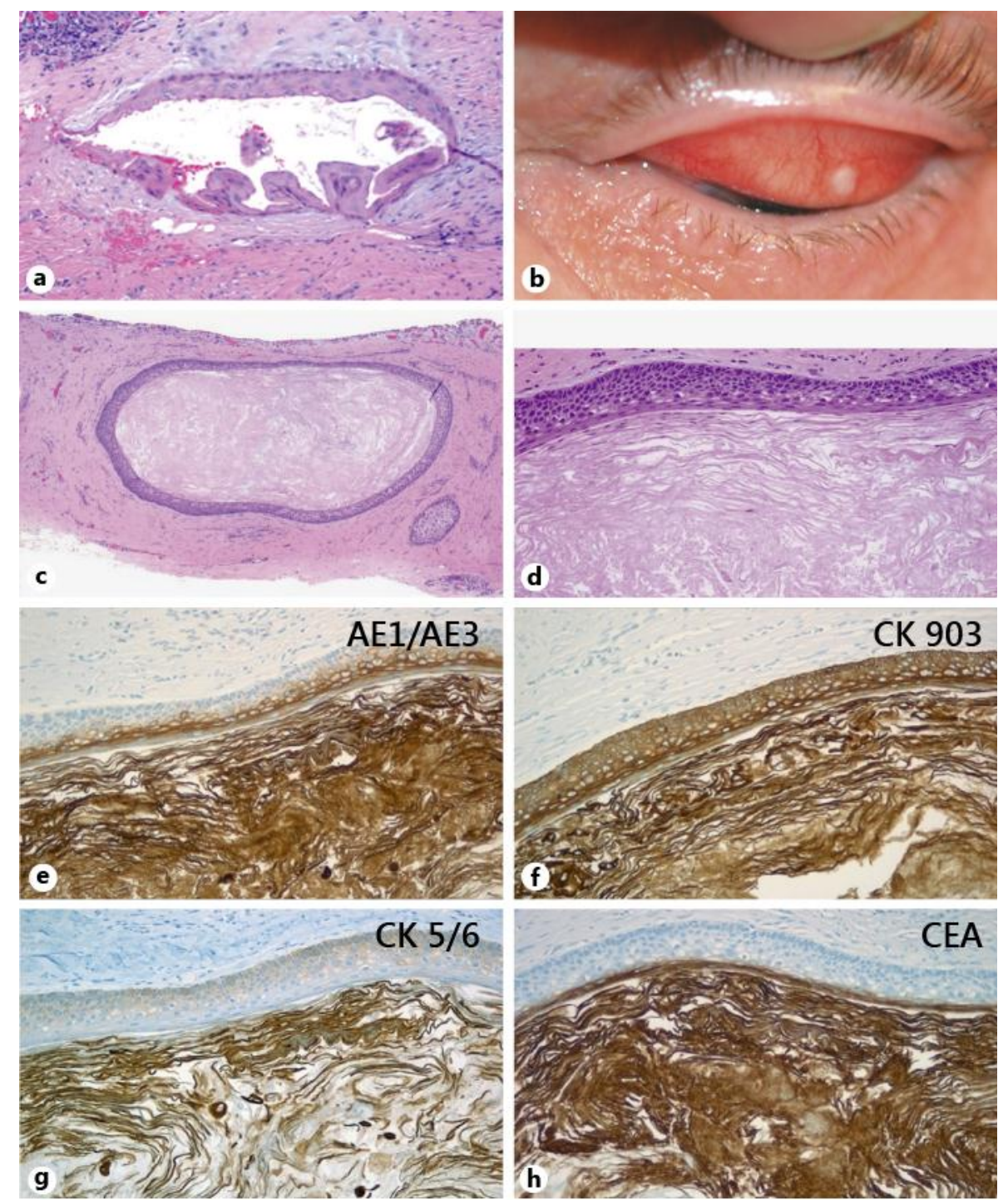

Fig. 1. Clinical image and histopathologic analysis of intratarsal keratinous cysts. a High-power view of the cyst described in case 1 stained with hematoxylin and eosin (H\&E). b Clinical photograph of the intratarsal keratinous cyst in the left upper eyelid described in case 2. c Low-power view of the cyst described in case 2, stained with H\&E, highlighting its intratarsal location. d Higher magnification view of the cyst in case 2, stained with H\&E. e AE1/AE3 is expressed in the apical epithelium as well as in the keratin filling. $\mathbf{f}$ CK 903 is expressed throughout the cyst epithelium and keratin. $\mathrm{g}$ CK 5/6 expression is faintly detected within the cyst epithelium and keratin. $\mathbf{h}$ CEA is expressed in the cyst cuticle and keratin. 